\title{
Adult $\alpha_{1}$-antitrypsin deficiency
}

\author{
E. C. SWEENEY
}

\begin{abstract}
From the Department of Histopathology, Royal Postgraduate Medical School, Hammersmith Hospital, Ducane Road, London W12
\end{abstract}

SYNOPSIS Three adults with $a_{1}$-antitrypsin deficiency are described. In two of the cases the deficiency was genetically determined (cases 1 and 2), and each demonstrated unusual features of the disease. The liver in case 1 (homozygous) showed cholangiolar hyperplasia which has been recorded only once before. Case 2 (heterozygous) had emphysema and cirrhosis, a combination not previously documented in a heterozygote, in addition to malabsorption. Case 3 represents a case of spurious $a_{1}$-antitrypsin deficiency with cirrhosis included to emphasize the diagnostic importance of phenotyping in such cases.

The rôle of $\alpha_{1}$-antitrypsin $\left(\alpha_{1} \mathrm{AT}\right)$ deficiency in the aetiology of early onset pulmonary emphysema and juvenile hepatic cirrhosis has been well established (Williams and Fajardo, 1974). Recent reports have shown that deficiency of this glycoprotein enzyme inhibitor may be associated with cirrhosis in older age groups also (eg, Ishak et al, 1972; Kumar et al, 1974). We present here the case histories of three adult patients with $\alpha_{1}$-AT deficiency which demonstrate unusual features of this disorder.

\section{Case Reports}

CASE 1

A 60-year-old woman gave a history of repeated episodes of 'bronchitis' as a child followed by a symptom-free adolescence and early adult life. She started smoking in late adolescence and smoked 10 cigarettes daily. Recurrent bronchitic episodes developed at the age of 40 years, and 10 years later she presented with severe breathlessness and purulent sputum. A plain radiograph of the chest showed cardiac enlargement with increase in the size of pulmonary arteries, a low flattened diaphragm, and emphysematous lung fields. Frequent bouts of chest infection accompanied by progressive deterioration in lung function occurred during the ensuing eight years, and ultimately she was admitted with respiratory failure and cor pulmonale. She improved with standard treatment but was noted to have a five-finger non-tender hepatomegaly. At the time of discharge from hospital her vital capacity was 2.051 , forced expired volume in one second 0.51 , and

Received for publication 2 January 1975.
$\mathrm{P}_{\mathrm{v}} \mathrm{CO}_{2} 58 \mathrm{mmHg}$. Her terminal admission was with acute on chronic respiratory failure. She was grossly cyanosed and hypercapnoeic $\left(\mathrm{P}_{\mathrm{v}} \mathrm{CO}_{2} 95 \mathrm{mmHg}\right)$ and demonstrated tender five-finger hepatomegaly. On admission respiratory arrest developed and intermittent positive pressure ventilation was begun. Culture of a bronchial aspirate yielded a growth of Staphylococcus aureus. The patient became clinically jaundiced with a serum bilirubin of $1.6 \mathrm{mg} \%$ and alkaline phosphatase of $14 \mathrm{King}$-Armstrong units (140 iu). There was a very low $\alpha_{1}$ globulin level and the serum $\alpha_{1}$-AT level was $0.23 \mathrm{~g} / \mathrm{l}$. The patient became unrousable and died.

\section{Necropsy}

The body was that of a slightly icteric elderly woman. The heart weighed $500 \mathrm{~g}$ and demonstrated severe right ventricular hypertrophy. There was diffuse centrilobular emphysema in both lungs and extensive atheroma in the pulmonary arteries. The liver weighed $1345 \mathrm{~g}$ and had smooth, yellowishbrown capsular and cut surfaces. The remainder of the postmortem examination was unremarkable. Microscopy of the lungs confirmed the presence of centrilobular emphysema. In the liver there was remarkable cholangiolar proliferation without evidence of bile stasis or cirrhosis (fig 1). Staining of diastase-treated sections by periodic-acid Schiff technique (SSD) revealed masses of globular intracytoplasmic inclusions in the hepatocytes mainly adjacent to the portal tracts (fig 2 ).

CASE 2

A 50-year-old man gave a history of chronic bronchitis from the age of $\mathbf{4 0}$ years. He smoked 10-15 
614
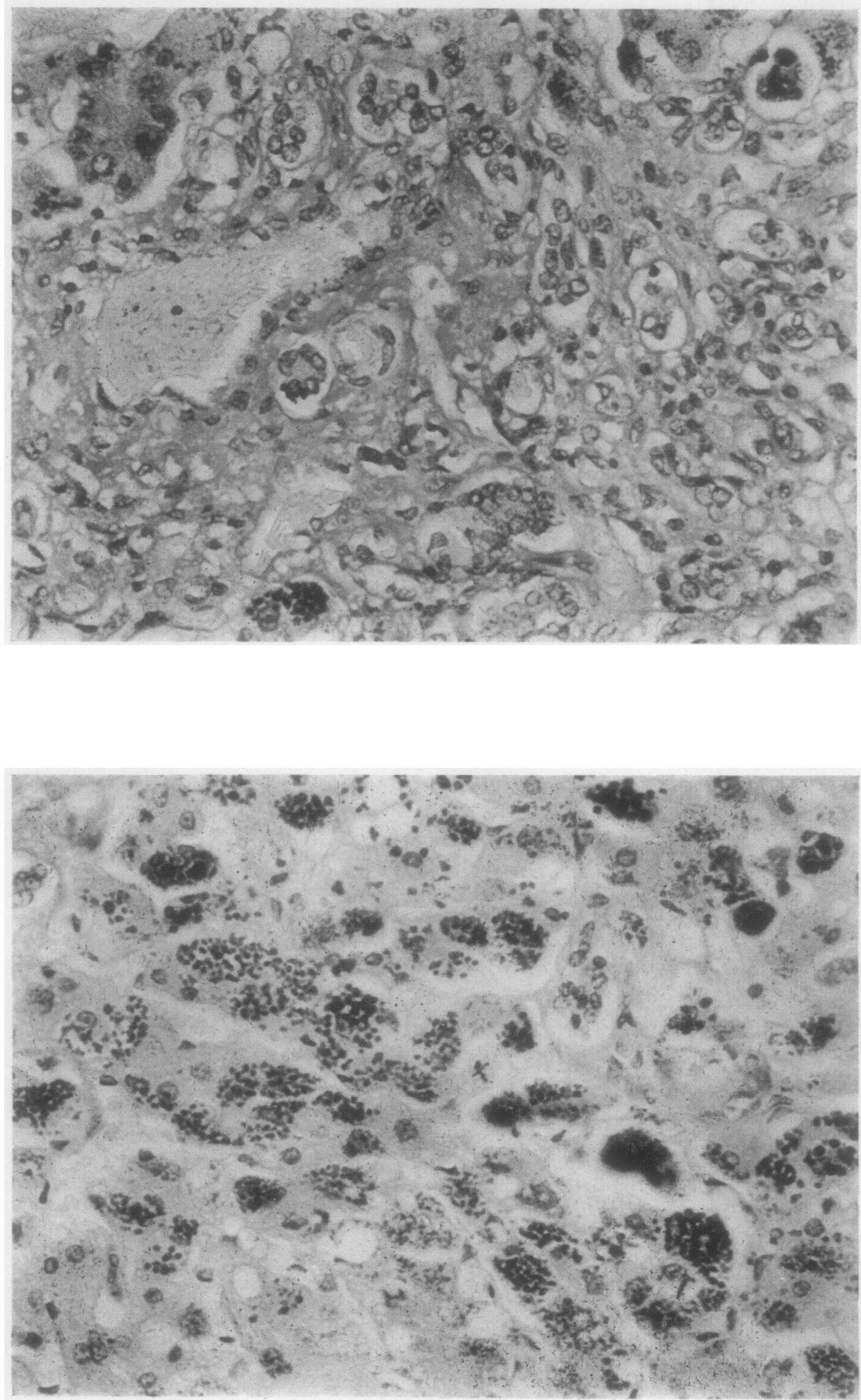

E. C. Sweeney

Fig 1 Case 1.

Demonstrating cholangiolar

hyperplasia.

$S S D \times 160$

(approx)

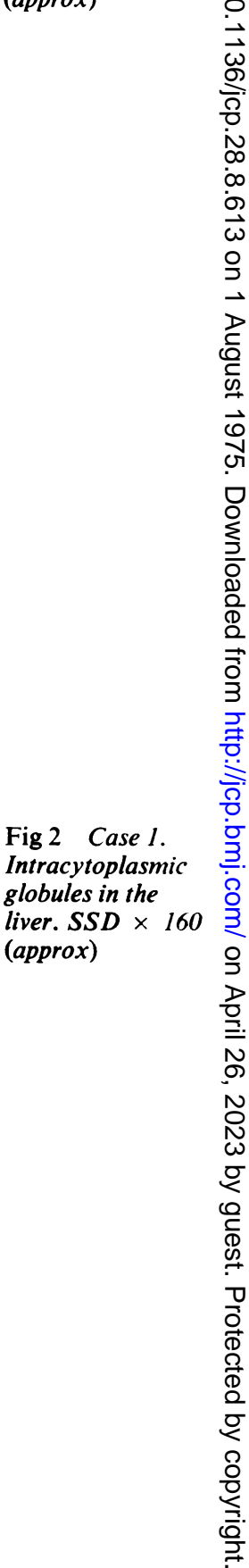




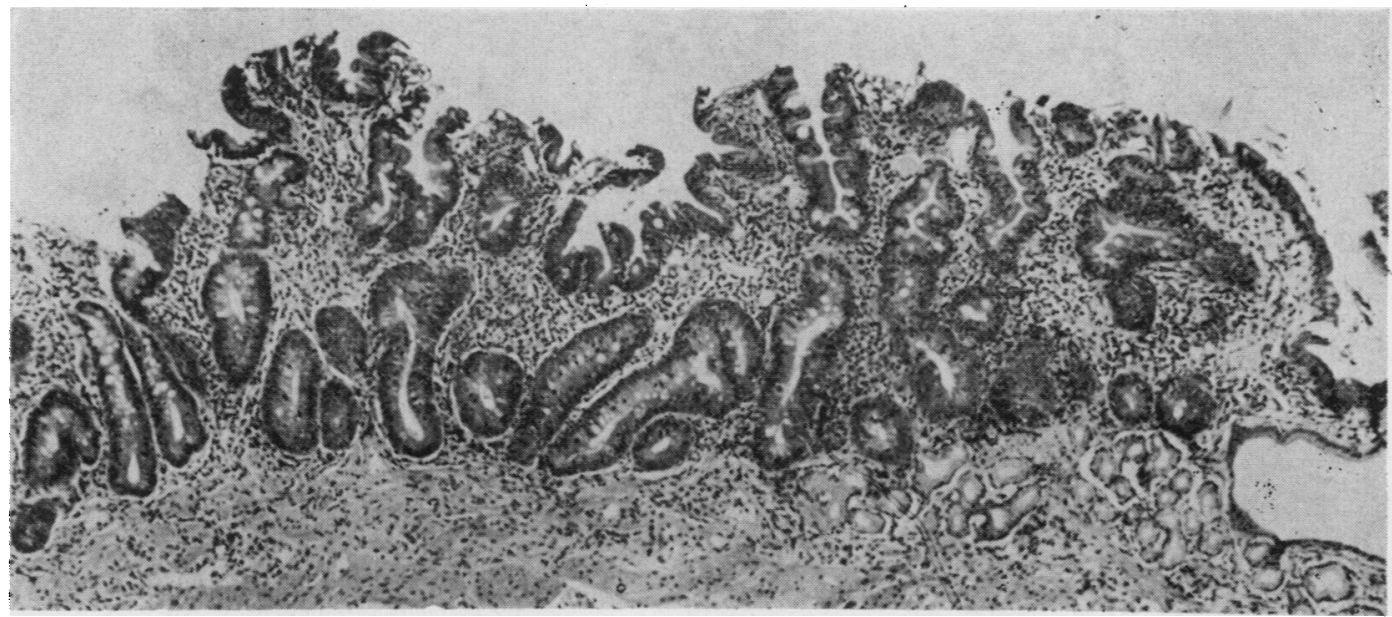

Fig 3 Case 2. Partial villous atrophy.

cigarettes and drank 3-4 pints of beer daily for many years. At the age of 48 years he had developed cor pulmonale. One year later steatorrhoea became manifest accompanied by weight loss and abdominal distension. After a further year the patient was admitted to hospital for investigation. Physical examination revealed clubbing of the fingers, hepatosplenomegaly, and evidence of emphysema. While in hospital he developed a left pneumothorax and a chest radiograph after drainage demonstrated bilateral basal bullous emphysema. A jejunal mucosal biopsy showed partial villous atrophy (fig 3). Faecal fat excretion was $60 \mathrm{~g} / 24$ hours but excretion of d-xylose was $5.4 \mathrm{~g}$ in 5 hours after a $25 \mathrm{~g}$ oral dose. A needle biopsy of the liver revealed some infiltration of the portal tracts by chronic inflammatory cells and accumulation of SSD positive globules within the hepatocytes (fig 4). Serum protein electrophoresis demonstrated a low albumin, a lack of rise in $\alpha_{2}$ globulin, and a raised $\gamma$ globulin, with $\beta / \gamma$ fusion, typical of cirrhosis. The $\alpha_{1}$ globulin was low, and measurement of $\alpha_{1}$-AT yielded a value of $1.2 \mathrm{~g} / \mathrm{l}$, suggesting that the patient was heterozygous for the deficiency. An attempt was later made to determine the patient's genotype but unfortunately the serum had deteriorated on storage. A barium meal and follow-through examination showed loss of mucosal pattern and dilatation of the upper jejunum with some thickening of the bowel wall and a small area of ulceration in this region. The patient was discharged on a gluten-free diet in addition to digoxin and diuretics. At subsequent reviews there was little change in the patient's jejunal biopsy or faecal fat excretion.

Two months after discharge the patient was readmitted with a perforation of the mid-jejunum. At laparotomy the liver was noted to be slightly enlarged and pale, with an irregular surface. The spleen was of approximately normal size. The small bowel was generally distended but there was a $30 \mathrm{~cm}$ long constriction in the mid to lower jejunum with a mottled greyish serosal surface. In the centre of this was a $3 \mathrm{~mm}$ perforation. Two similar but shorter constricted segments were present elsewhere in the small bowel. The perforation was excised. Postoperatively the patient developed irreversible cardiac arrest.

\section{Necropsy}

The body was that of a wasted middle-aged man. There was widespread centrilobular emphysema, more marked in both lower lobes. The heart weighed $350 \mathrm{~g}$ and demonstrated moderate right atrial and right ventricular dilatation. In the abdomen the loops of small bowel were loosely adherent and covered with fibrinous exudate. The mucosa of the proximal jejunum was flattened, and at the jejunoileal junction there was a $12 \mathrm{~cm}$ long stricture showing the site of the previous perforation covered by an intact patch. Similar shorter constricted areas were present in the ileum. In these areas the bowel lumen was reduced to approximately one-half without thickening of the wall. Microscopy of the stenotic zones showed mucosal ulceration with massive submucosal infiltration by lymphocytes and plasma cells and formation of numerous lymphoid follicles. The muscularis propria was thickened. The liver weighed $2875 \mathrm{~g}$ and showed fine irregular nodularity of the capsular surface with a coarse trabecular pattern on the cut surface. Microscopy demonstrated portal fibrosis with prominent bile duct reduplication surrounding hyperplastic nodules 


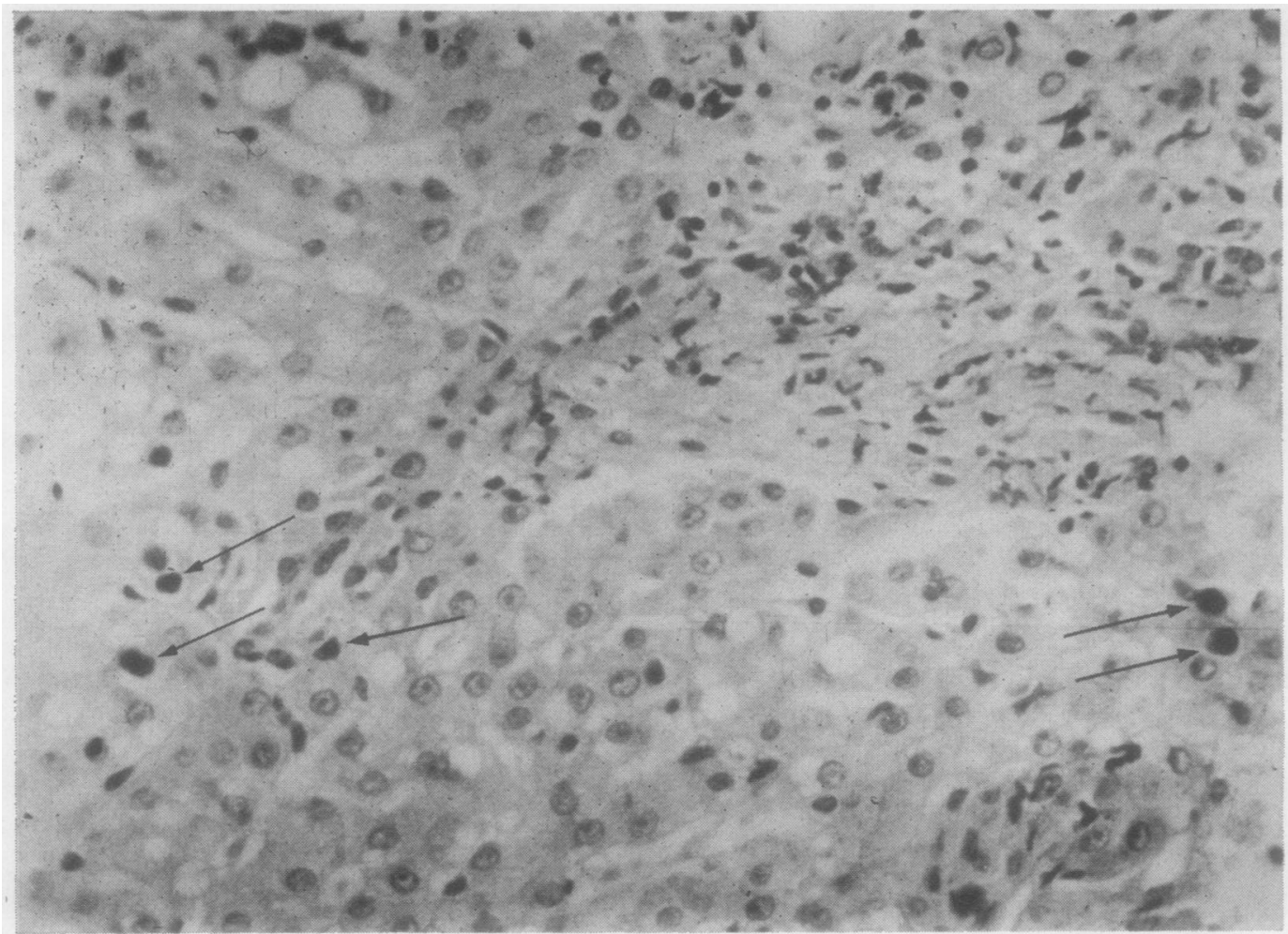

Fig 4 Case 2. Intracytoplasmic globules (arrowed) in liver. SSD $\times 200$ (approx)

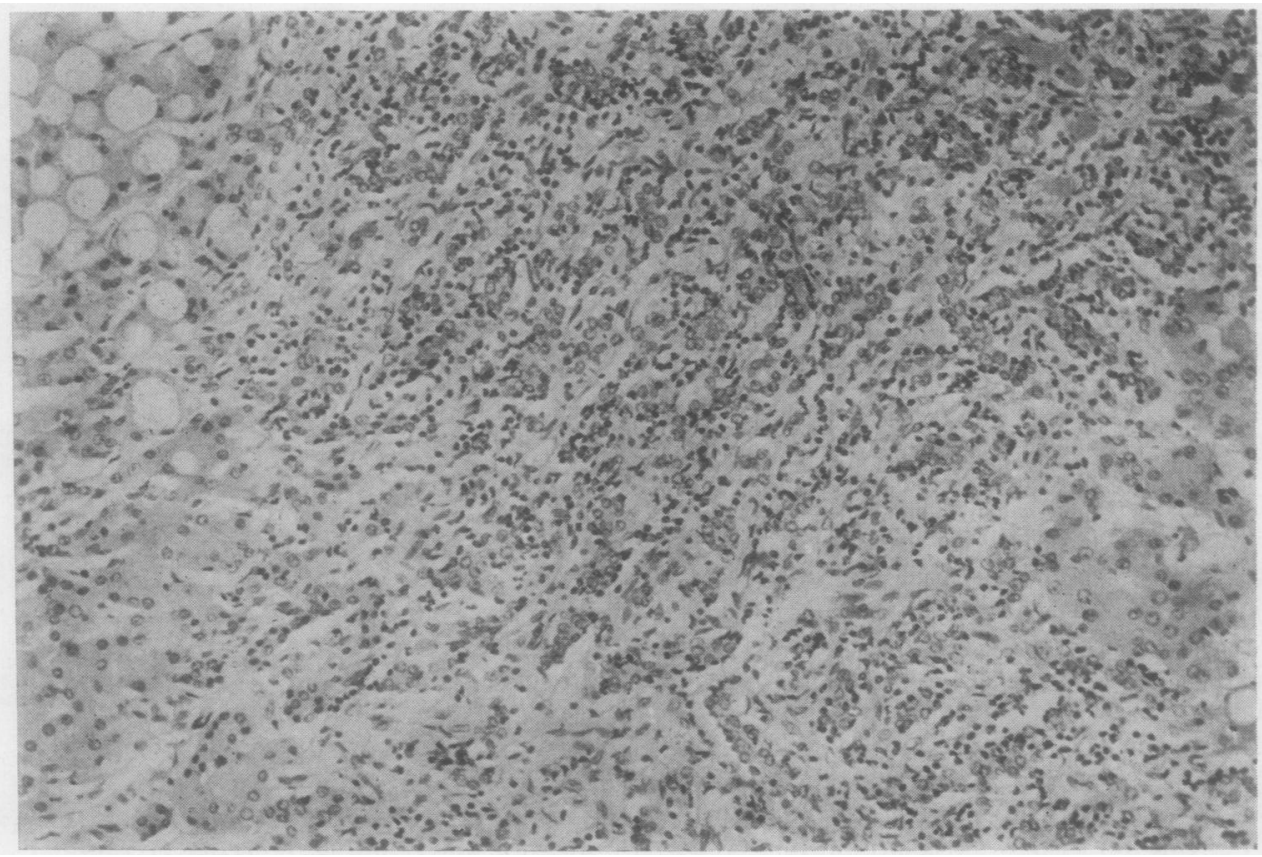

Fig 5

Case 2.

Demon-

strating pro-

liferation of

the small

bile ducts

in the liver.

$H$ and $E \times$

120 
of hepatocytes (fig 5). Examination of multiple levels of this tissue failed to reveal any of the SSD positive material which had been seen on biopsy.

\section{CASE 3}

A 53-year-old man gave a history of one episode of malaise and loin pain with dysuria at the age of 40 . He was asymptomatic for a further six years and was then hospitalized for investigation of nausea. Chronic renal disease with hypertension was diagnosed and the patient was treated with dietary restriction and antihypertensive drugs.

At the age of 50 years the patient was admitted for treatment of renal failure. Renal transplantation was not feasible because of donor incompatibility, and long-term haemodialysis was started. During the next three years the patient remained well but intractable hypochromic anaemia necessitated frequent transfusions (214 units over three years). Serum protein estimation before one of these transfusions showed a total of $6 \cdot 1 \mathrm{~g} / 100 \mathrm{ml}$ with an albumin of 3.5 and a globulin of $2 \cdot 6$. Electrophoresis revealed a low $\alpha_{1}$ globulin and the estimated level of $\alpha_{1}$-AT was $40-44 \mathrm{mg} / 100 \mathrm{ml}$. However, proteinuria

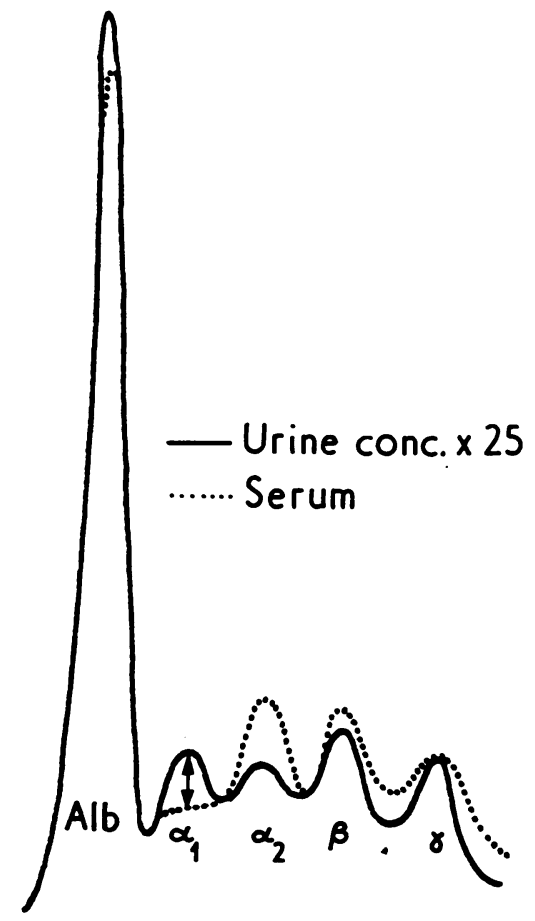

Fig 6 Case 3. Superimposed urine and serum protein electrophoretic graphs. Note the disparity between the $\alpha_{1}$ globulin levels. was present $(110 \mathrm{mg} / 100 \mathrm{ml})$ and concentrated urine electrophoresis revealed a non-selective pattern with an excess of $\alpha_{2}, \gamma$ and even $\beta_{2}$ compatible with severe proliferative glomerulonephritis (fig 6).

Skin pigmentation, testicular atrophy, and gynaecomastia gradually developed, suggesting progressive liver disease. A liver biopsy revealed increased amounts of fibrous tissue in the portal tracts and an abundance of haemosiderin pigment in both Kupffer and parenchymal cells. No SSD positive material was identified in the hepatocytes.

The arteriovenous shunt that had been inserted for haemodialysis became infected with Pseudomonas aeruginosa, and bacteraemia resulted in bacterial endocarditis and perforation of a mitral valve cusp. Replacement of the mitral valve was performed using a Starr-Edwards prosthesis but the patient suffered irreversible postoperative circulatory collapse and died.

\section{Necropsy}

The body was that of a thin pigmented middle-aged man with gynaecomastia and small atrophic testes. The heart weighed $570 \mathrm{~g}$ and showed moderate left ventricular hypertrophy. The mitral valve had been replaced by a prosthesis. The remaining valves were normal. Both lungs were oedematous but no emphysema was present. The liver weighed $2746 \mathrm{~g}$ and had a uniform yellowish colour with a strong positive staining reaction for iron on the cut surface. Both kidneys were small, each measuring $7.5 \times 4 \times$ $1.8 \mathrm{~cm}$ with numerous cortical cysts measuring $0.2-2 \mathrm{~cm}$ in diameter and an indistinct corticomedullary demarcation. The remainder of the necropsy was unremarkable. Microscopically the liver showed essentially the same features as the previous biopsy but with hyperplasia of the parenchymal cells in the centre of the nodules. Again no SSD positive material was seen. Massive amounts of iron pigment were present not only in the liver but also in the spleen, lymph nodes, pancreas, and thyroid. The kidneys demonstrated gross reduction in the number of glomeruli but those which remained appeared normal. A minor degree of tubular atrophy was present also. The appearances were consistent with a longstanding focal glomerulitis.

\section{Discussion}

The low molecular weight glycoprotein $\alpha_{1}-\mathrm{AT}$ is synthesized in the liver and has the capacity to inhibit a variety of proteolytic enzymes. Different types of $\alpha_{1}$-AT exist which can be separated on the basis of their electrophoretic mobility. Twenty alleles constitute the Pi system (Fagerhol, 1971; Cook, 1974). The common genotype giving normal 
levels of $\alpha_{1}$-AT (1.80 to $\left.5.00 \mathrm{~g} / \mathrm{l}\right)$ is PiMM whereas homozygously deficient individuals have the genotype PiZZ. In the latter cases the serum level of $\alpha_{1}-\mathrm{AT}$ is approximately $10 \%$ that of normal (less than 0.50 $\mathrm{g} / \mathbf{l}$ ). Intermediate levels are found in heterozygotes, in association with the $\mathrm{Pi}^{\mathrm{s}}$ and $\mathrm{Pi}^{\mathrm{p}}$ alleles, and zero levels may be found with the $\mathrm{Pi}^{\text {null }}$ allele (Talamo et al, 1973). Homozygous (PiZZ) individuals constitute approximately $0 \cdot 1-0.2 \%$ of the general population whereas heterozygotes (PiSZ, PiMZ, PiMS, etc) constitute approximately $12 \%$.

The diseases which have been associated with a deficiency of $\alpha_{1}-\mathrm{AT}$ are principally pulmonary emphysema of early onset (Eriksson, 1964) and juvenile hepatic cirrhosis (Sharp et al, 1969). It has been estimated that two-thirds of individuals homozygous for the deficiency will develop emphysema (Eriksson, 1965) but the reason why the remaining third do not develop it is obscure. Cirrhosis occurring in adult homozygous individuals has been described both with (Berg and Eriksson, 1972; Babb et al, 1973; Cohen et al, 1973) and without (Ishak et al, 1972; Kumar et al, 1974) emphysema. In heterozygous individuals emphysema does occur (Kanner et al, 1973; Stevens et al, 1971) and cirrhosis has also been described in two heterozygotes (Campra et al, 1973; Brand et al, 1974), but the combination of cirrhosis and emphysema in the heterozygous state has not been reported before. In the livers of all homozygously deficient individuals (Berg and Eriksson, 1972) the hepatocytes contain globular SSD positive intracytoplasmic masses of material antigenically similar to $\alpha_{1}$-AT. This suggests that release of the inhibitor from the cells is impaired and that this might in some way be due to abnormal molecular configuration of the variant antienzyme (Lieberman et al, 1972). The same authors and others (Gordon et al, 1972) also found intracytoplasmic globules in the livers of heterozygous patients with emphysema. The histological features of cirrhosis occurring in $\alpha_{1}$-AT deficiency are distinguishable from other forms of cirrhosis only by these intracytoplasmic globules.

Various facets of the three cases reported here are of interest :

\section{CASE 1}

This patient presented as a classical case of $\alpha_{1}-\mathrm{AT}$ deficiency with emphysema and developed clinical evidence of liver disease only terminally. The extremely low level of $\alpha_{1}$-AT in the serum indicated that the patient was homozygous for the deficiency, and the finding of intracytoplasmic globules in the liver was not unexpected. However, the histology of the liver was unusual, showing as it did pronounced cholangiolar hyperplasia without portal fibrosis or bile stasis. Such an appearance has been described $\stackrel{.}{\Rightarrow}$ only once before (Gherardi, 1971) also in a homo- $\stackrel{\vec{S}}{\rightarrow}$ zygously deficient individual, but in that case the $\overline{0}$ presence of intracytoplasmic globules was not 흠 recorded. Whether this represents an early stage in $\frac{\bar{\rho}}{\overrightarrow{ }}$ the development of cirrhosis or an alternative $\stackrel{\mathbb{\Phi}}{\propto}$ reaction to injury in this deficiency disease remains conjectural.

\section{CASE 2}

The initial development in this patient was empiny- $\stackrel{\omega}{\sigma}$ sema. The aetiology of the malabsorption that subsequently developed is obscure as is its relation- i ship to the $\alpha_{1}$-AT deficiency. The biochemical and $\underset{\infty}{\infty}$ histological evidence argues in favour of it being $\sigma$ adult coeliac disease in which condition stricture $\vec{\omega}$ formation and perforation of the small bowel have 의 been recorded before (Bayless et al, 1967). It was unfortunate that the genotype of this patient could $\stackrel{\square}{\perp}$ not be determined, but judging by the serum $\alpha_{1}$ 읃 antitrypsin level he was heterozygous for the $\stackrel{\oplus}{+}$ deficiency and probably represents the first hetero- $\vec{\theta}$ zygous case in which both emphysema and cirrhosis of have been recorded. A point of interest is the discrepancy between the amount of intracytoplasmic material seen in the initial needle biopsy and the lack of it in the postmortem material. This is at variance with the findings of other authors (Gordon $\frac{2}{\varnothing}$ et al, 1972) who noted that necropsy specimens of $\stackrel{\square}{\vec{Q}}$ liver seemed to contain more $\alpha_{1}$-AT globules than $\overrightarrow{\bar{O}}$ biopsy specimens. They attributed this to $\alpha_{1}-\mathrm{AT}$ being an acute phase reactant and postulated that it is produced and (in its abnormal forms) accumulates to a greater extent in acutely ill patients.

\section{CASE 3}

This patient has been included in this report as a 0 reminder that all that glitters is not gold. The low serum $\alpha_{1}$-AT level which fell within the homozygous 의 range was not due to an inherent deficiency but to $\rightarrow$ loss of the glycoprotein in the urine. Had this been a true case of $\alpha_{1}$-AT deficiency then the typical SSD positive globules would have been present in the liver. The hepatic cirrhosis was entirely explicable $N$ on the basis of excessive iron deposition.

To date four cases of renal disease have been recorded in association with $\alpha_{1}$-AT deficiency (Miller 0 and Kuschner, 1969; Hobbs, 1971). The case of $\frac{\complement}{\mathbb{}}$ Miller and Kuschner appears to have been a genuine $\stackrel{\oplus}{?}$ homozygously deficient individual who developed $T$ emphysema and some years later a necrotizing $\stackrel{\vec{P}}{\vec{P}}$ arteritis and glomerulonephritis with massive $\stackrel{\odot}{\stackrel{\odot}{\circ}}$ proteinuria. Strangely, the characteristic intra- $\stackrel{\mathbb{Q}}{\varrho}$ cytoplasmic hepatic globules were not seen. To date $\frac{\sigma}{\sigma}$ the three cases recorded by Hobbs have not been described in sufficient detail to compare with ouro 
case. Case 3 highlights the need for phenotyping and exclusion of protein loss in all apparent cases of $\alpha_{1}$-AT deficiency.

My thanks are due to Dr D. J. Evans for his help in producing this paper.

\section{References}

Babb, R. R., Lillington, G. A., and Kempson, R. L. (1973). Cirrhosis in an adult with emphysema and alpha-l-antitrypsin deficiency. Amer. J. dig. Dis., 18, 803-807.

Bayless, T. M., Kapelowitz, R. F., Shelley, W. M., Ballinger, W. F., II., and Hendrix, T. R. (1967). Intestinal ulceration: a complication of coeliac disease. New Engl.J. Med., 276, 996-1002.

Berg, N. O. and Eriksson, S. (1972). Liver disease in adults with alpha $\mathrm{a}_{1}$-antitrypsin deficiency. New Engl. J. Med., 287, 25, 1264-1267.

Brand, B., Bezahler, G. H., and Gould, R. (1974). Cirrhosis and heterozygous $\mathrm{FZ} \alpha_{1}$ antitrypsin deficiency in an adult. Gastroenterology, 66, 264-268.

Campra, J. L., Craig, J. R., Peters, R. L., and Reynolds, T. B. (1973). Cirrhosis associated with partial deficiency of alpha-1-antitrypsin in an adult. Ann. intern.Med., 78, 233-238.

Cohen, K. L., Rubin, P. E., Echevarria, R. A., Sharp, H. L., and Teague, P. O. (1973). Alpha-l-antitrypsin deficiency, emphysema and cirrhosis in an adult. Ann. intern. Med., 78, 227-232.

Cook, P. J. L. (1974). Genetic aspects of the Pi system. Postgrad. med. J., 50, 362-364.

Eriksson, S. (1964). Pulmonary emphysema and alpha-1antitrypsin deficiency. Acta med. scand., 175, 197-205.

Eriksson, S. (1965). Studies in alpha-1-antitrypsin deficiency. Acta med. scand., Suppl., 432.

Fagerhol, M. (1971). Le système des $\mathrm{Pi}$, sa génétique et ses rapports avec les maladies. Poumon, 27, 41-51.

Gherardi, G. J. (1971). Alpha-1-antitrypsin deficiency and its effect on the liver. Hum. Path., 2, 173-175.

Gordon, H. W., Dixon, J., Rogers, J. C., Mittman, C., and

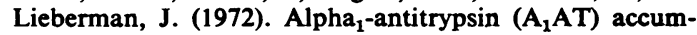
ulation in livers of emphysematous patients with $A_{1}$ AT deficiency. Hum. Path., 3, 361-370.

Hobbs, J. R. (1971). Deficiency of alpha-1-antitrypsin (abstract). J. clin. Path., 24, 482.

Ishak, K. G., Jenis, E. H., Marshall, M. L., Bolton, B. H., and Battistone, G. C. (1972). Cirrhosis of the liver associated with $\alpha_{1}$-antitrypsin deficiency. Arch. Path., 94, 445-455.

Kanner, R. E., Klauber, M. R., Watanabe, S., and Bigler, A. (1973). Pathologic patterns of chronic obstructive pulmonary disease in patients with normal and deficient levels of alpha-l-antitrypsin. Amer. J. Med., 54, 706-712.

Kumar, P., Lancaster-Smith, M., Cook, P., Stansfeld, A., Clark, M. L., and Dawson, A. M. (1974). $\alpha_{1}$ Antitrypsin deficiency in chronic liver disease, and a report of cirrhosis and emphysema in adult members of a family. Brit. med.J., 1, 366-377.

Lieberman, J., Mittman, C., and Gordon, H. W. (1972). Alpha-1-antitrypsin in the livers of patients with emphysema. Science, 175, 63-i5.

Miller, F. and Kuschner, M. (1969). Alpha $a_{1}$-antitrypsin deficiency, emphysema, necrotizing angiitis and glomerulonephritis. Amer. J. Med., 46, 615-623.

Sharp, H. L., Bridges, R. A., Krivit, W., and Freier, E. F. (1969). Cirrhosis associated with alpha-1-antitrypsin deficiency: a previously unrecognised inherited disorder. J. Lab. clin. Med., 73, 934-939.

Stevens, P. M., Hnilica, V. S., Johnson, P. C., and Bell, R. L. (1971). Pathophysiology of hereditary emphysema. Ann. intern. Med., 74, 672-680.

Talamo, R. C., Langley, C. E., Reed, C. E., and Makino, S. (1973). Alpha-l-antitrypsin deficiency: A variant with no detectable alpha-1-antitrypsin. Science, 181, 70-71.

Williams, W. D. and Fajardo, L. F. (1974). Alpha-l-antitrypsin deficiency: a hereditary enigma. Amer. J. clin. Path., 61, 311-320.

\section{Addendum}

Since this paper was submitted for publication a case of adult $\alpha_{1}$-antitrypsin deficiency with intestinal mucosal atrophy, cirrhosis, and emphysema has been reported (Greenwald, A. J., Johnson, D. S., Oskvig, R. M., Aschenbrener, C. A., and Randa, D. C., $\alpha_{1^{-}}$ Antitrypsin deficiency, emphysema, cirrhosis and intestinal mucosal atrophy (1975) J. Amer. med. Ass., 231,$3 ; 273$ ). The patient had a severe deficiency of the antienzyme $(50 \mathrm{mg} / 100 \mathrm{ml})$ and gluten sensitive 'coeliac-like' disease. The authors speculate that the association is more than fortuitous. 